Article

\title{
Liquid-Based Screening Tests Results: HPV, Liquid-Based Cytology, and P16/Ki67 Dual-Staining in Private-Based Opportunistic Cervical Cancer Screening
}

\author{
Martyna Trzeszcz 1,2,*(D), Maciej Mazurec 1,*(D), Robert Jach ${ }^{3,4}$, Karolina Mazurec ${ }^{1}$, Zofia Jach ${ }^{4}$, \\ Izabela Kotkowska-Szeps ${ }^{1}$, Magdalena Kania ${ }^{1}$, Mariola Wantuchowicz ${ }^{1}$, Anna Prokopyk ${ }^{1}$, Piotr Barcikowski ${ }^{1}$, \\ Marcin Przybylski ${ }^{5}$, Joanna Wach ${ }^{1,6}$ and Agnieszka Halon ${ }^{7}$
}

check for updates

Citation: Trzeszcz, M.; Mazurec, M.; Jach, R.; Mazurec, K.; Jach, Z.; Kotkowska-Szeps, I.; Kania, M.; Wantuchowicz, M.; Prokopyk, A.; Barcikowski, P.; et al. Liquid-Based Screening Tests Results: HPV, LiquidBased Cytology, and P16/Ki67 Dual-Staining in Private-Based Opportunistic Cervical Cancer Screening. Diagnostics 2021, 11, 1420. https: / / doi.org/10.3390/ diagnostics 11081420

Academic Editor: Ivana Kholová

Received: 18 July 2021

Accepted: 2 August 2021

Published: 5 August 2021

Publisher's Note: MDPI stays neutral with regard to jurisdictional claims in published maps and institutional affiliations.

Copyright: (C) 2021 by the authors Licensee MDPI, Basel, Switzerland. This article is an open access article distributed under the terms and conditions of the Creative Commons Attribution (CC BY) license (https:/ / creativecommons.org/licenses/by/ $4.0 /)$.
1 Corfamed Woman's Health Center, Kluczborska 37, 50-322 Wroclaw, Poland; carlos.mazurec@icloud.com (K.M.); iza.corfamed@gmail.com (I.K.-S.); magda_kania@poczta.onet.pl (M.K.); mariolawantuchowicz@gmail.com (M.W.); ania.prokopyk@gmail.com (A.P.); barcikowski07@gmail.com (P.B.); asia03529@gmail.com (J.W.)

2 Division of Pathology and Clinical Cytology, University Hospital in Wroclaw, Borowska 213, 50-556 Wroclaw, Poland

3 Division of Gynecologic Endocrinology, Jagiellonian University Medical College, Kopernika 23, 31-501 Krakow, Poland; jach@cm-uj.krakow.pl

4 Superior Medical Center, Czyzynska 21/50, 31-571 Krakow, Poland; zofiajach@gmail.com

5 Department of Obstetrics and Gynecology, District Public Hospital, Juraszow 7-19, 60-479 Poznan, Poland; nicramp@poczta.onet.pl

6 Department of Practical Obstetrics, Wroclaw Medical University, Bartla 5, 51-618 Wroclaw, Poland

7 Department of Clinical and Experimental Pathology, Division of Clinical Pathology, Wroclaw Medical University, Borowska 213, 50-556 Wroclaw, Poland; agnieszka.halon@umed.wroc.pl

* Correspondence: m.trzeszcz@corfamed.pl (M.T.); m.mazurec@corfamed.pl (M.M.); Tel.: +48-508-646-805 (M.T.)

Abstract: The baseline data from the private-based opportunistic cervical cancer screening with HRHPV14, liquid-based cytology (LBC) and p16/Ki67 testing, and its quality assessment/quality control (QA/QC) tools are lacking. The age-stratified analysis of 30,066 screening tests results in a Polish population, including the investigation of HRHPV14 status, LBC, and p16/Ki67 dualstaining reporting rates, along with immediate histopathologic correlations, was conducted. For cytopathologic QA/QC, the College of American Pathologists (CAP) benchmarks and enhanced safety protocol were used. The NILM/ASC-US/LSIL/ASC-H/HSIL/AGC reporting rates were 93.9/3.4/2.0/0.22/0.24/0.11, respectively, with correlating HRHPV14-positive rates of 8.4/48.9/77.2/ 84.6/90.7/26.7. The reporting rates for HSIL (CIN2+) in HRHPV-positive women with NILM/ASCUS/LSIL/ASC-H/HSIL/AGC referred for a colposcopy with biopsy were 19.1/25.8/22.5/12.4/19.1/ $1.1 \%$ of the total HSIL (CIN2+). In total, of the $1130 \mathrm{p} 16 / \mathrm{Ki} 67$ tests, $30 \%$ were positive. In NILM HRHPV14-positive women with available histology result, HSIL(CIN2+) was detected in $28.3 \%$ of cases. In the first such large-scale Polish study presenting HRHPV14, informed LBC and HSIL (CIN2+) results, the reporting rates were highly consistent with data from American and other CAPcertified laboratories, confirming the possibility of using the 2019 ASCCP risk-based guidelines as one of the screening strategies outside of the US, in conditions of proper QA/QC. The private-based screening model can be effective in cervical cancer prevention, particularly in countries with low population coverage of public funds-based systems.

Keywords: cervical cancer screening; high-risk HPV testing; cervical cytology; p16/Ki67 dualstaining; prevention and control

\section{Introduction}

HPV testing is globally recommended as a primary screening strategy in cervical cancer prevention in all resource settings [1]. According to the European guidelines, it 
should be implemented under the population-based organized system, which is currently the most effective model of screening [2].

In Poland, a Central Eastern Europe country, two main models of cervical cancer screening have been coexisting for years: a public funds-based and a private-based model. In the years 2006-2016, the only organized population-based national program with primary cytology screening in 3-year screening intervals was implemented [3-5]. However, coverage of the target group in the program did not exceed 27\% [6,7], which does not meet the recommended $70 \%$ population coverage needed to reach screening effectiveness [2]. In parallel to the public funds-based screening in Poland, there is a private-based screening financed directly by the patients. Its exact screening coverage remains unknown, but published questionnaire data suggest a significant percentage of involvement. In 2012, the participation of Polish women in screening outside of the program was 1.7 times higher than in the program $[4,8]$, and in the last 3 years, about $70 \%$ of women have had a Pap test [9], which clearly suggests the dominating role of the private-based screening. There are various models in Polish private-based screening, from cytology to cotesting; the latter was approved in 2013 [10].

Due to the high sensitivity and high specificity of p16/Ki67 dual-staining for the detection of HSIL (CIN2+) [11-13], it was introduced in Poland for triaging patients with ASC-US or LSIL in 2016 [14]. The effectiveness of the extended screening strategy in the HPV-based model with p16/Ki67 testing has been confirmed by FDA registration [15]. The limitations of the Polish population-based screening, the preferences of Polish women, and the growing awareness of private sector gynecologists in the new paradigms of secondary cervical cancer prevention result in the gradual implementation of the globally recommended HPV-based screening strategies with proven higher effectiveness $[2,16]$ in Polish private gynecological care $[17,18]$. Interim screening guidelines in the SARS-CoV-2 pandemic of 2021 [18] include the 2019 risk-based management guidelines of the American Society of Colposcopy and Cervical Pathology (ASCCP) [16] as one of the screening options. The 2019 ASCCP guidelines indicate that women with the same screening tests results, together with a similar screening history, have a similar CIN3+ risk, regardless of geographic location, race, ethnicity, or socioeconomic status.

We conducted a retrospective analysis of liquid-based screening (LBS) tests results, including liquid-based cytology (LBC), 14 types of high-risk human papilloma virus (HRHPV14), and p16/Ki67 HSIL-risk biomarkers, along with histologic correlations in private-based opportunistic cervical cancer screening. This is the first Polish investigation on such a large scale. The second objective of the study was a comparative analysis of the obtained cytologic and virologic reporting rates with the American data for gynecological cytopathology, in order to verify the effectiveness of the quality assessment and quality control (QA/QC) tools used, confirming that it is possible to apply the 2019 ASCCP guidelines in private-based opportunistic screening outside the US. Our study also addresses how to open the way for an integration of organized population-based and opportunistic private-based models into a co-existing hybrid model, giving the opportunity to achieve the required population screening coverage in Poland and in other countries with similar economic-cultural conditions.

\section{Materials and Methods}

\subsection{Study Population}

This study is a post-hoc analysis on LBS, including LBC, HRHPV14, and p16/Ki67 tests results, obtained in private-based opportunistic screening in one of the largest private outpatient gynecological clinics in an urban area of the Lower Silesia in Poland (Corfamed Woman's Health Center) from August 2015 to July 2020. This age-stratified analysis comprises a total of 30.066 screening tests. Three cervical cancer screening models have been applied in the Center during the analyzed period: primary cytology with reflex HPV testing, primary cotesting including simultaneous performance of HPV testing and cytology, and primary cotesting plus (cotesting with simultaneous p16/Ki67 testing). 
In the primary cytology screening, the reflex HRHPV14 test was recommended in all cases with minor cytological abnormalities (ASC-US or LSIL). The p16/Ki67 test was performed in cytology- and cotesting-based models, in cases with ASC-US or LSIL in cytology, and for all positive HRHPV14 test results. The retrospective analysis of cytologicvirologic-immunocytochemical tests results, along with the histology results obtained from colposcopies performed at the Center, were the endpoints of this study. All analyzed data were from the Centre's registry.

\subsection{HRHPV Testing}

For HRHPV detection, the Abbott RealTime High Risk HPV molecular in vitro PCR test was used. A laboratory performing the test declared that it was in accordance with the manufacturer's protocol. The test phenotypes the DNA of 14 highly oncogenic HPV types $(16,18,31,33,35,39,45,51,52,56,58,59,66$, and 68) with HPV 16 and 18 genotyping. All screening tests (HRHPV14, LBC and p16/Ki67) were performed in the same laboratory.

\subsection{Cytology}

All cervical cytology was collected by the Center's gynecologists using the CervexBrush device (Rovers Medical Devices, Oss, The Netherlands) and transferred on the detachable head into the SurePath vial containing a preservative fluid. Cervical sampling was carried out with rotation of the broom in a clockwise direction five times, after inserting the central bristles into the endocervical canal. The SurePath liquid-based preparation was performed in accordance with the automated PrepStain procedure (Becton Dickinson, Franklin Lakes, NJ, USA) by an external laboratory, which ensured that its performance strictly adhered to a manufacturer's protocol. All cytology samples were reported by a gynecological cytopathologist in the Bethesda 2014 system. A pathologist was employed by the Center and was informed about HRHPV14 status. All residual cervical samples were stored for up to 6 months by the laboratory under the conditions specified by the manufacturer. It provided no need to call the patient for another visit when an ancillary testing was ordered (including the p16/Ki67).

\subsection{P16/Ki67 Dual-Stain Testing}

For immunocytochemical double staining with p16 and Ki67 proteins, a CINtec PLUS detection kit (Roche, MTM AG laboratories, Munich, Germany) was used and carried out in an automated BenchMark XT system (Ventana Medical Systems, Inc., Oro Valley, AZ, USA) according to the manufacturer's protocol, as provided by the coordinator from the laboratory. The p16/Ki67 test was performed from the residual LBC material in the original SurePath vials (Becton Dickinson, Franklin Lakes, NY, USA), stored in a laboratory after cytology and/or HPV testing. The p16/Ki67 tests were evaluated by a gynecological pathologist (the same pathologist as for the LBC evaluation), specially trained and certified in p16/Ki67 test review, and reported as positive/negative/unsatisfactory in accordance with the available definitions [19].

\subsection{Basic and Extended QA/QC Protocols}

Gynecologic cytopathology QA/QC tools for morphologic evaluation in LBC preparation have not yet been standardized in Polish conditions, hence the need for the Center to rely on the available CAP QA/QC protocols. We modified the CAP protocol due to the p16/Ki67 testing used in this study, and the Center's own original protocol for LBS tests results, including LBC, p16/Ki67 and HRHPV14, was created. Two protocols have been standardized in the study: (1) basic, the continuous quality improvement (CQI) protocol; (2) extended, the enhanced safety (ES) protocol. The basic protocols were used for the entire study group of 20,605 LBC, and the extended protocol for was used for the first 8330 LBC diagnoses. 


\subsubsection{QA/QC Basic Protocol}

In the internal QA/QC for LBC, a re-evaluation of $100 \%$ of ASC-US+ results (ASC-US or worse) and $100 \%$ of HRHPV14-positive NILM cases was performed, as well as ASC-H, HSIL/HRHPV14-negative, and NILM HRHPV14 type 16 and/or 18-positive cases. The re-evaluation of the LBC diagnoses was performed by one cytopathologist, the initial LBC results were blinded. Additionally, the CAP QA/QC gynecological cytopathology tools were implemented. For this purpose, obtained percentage rates in individual categories of cytological diagnoses were compared to the ranges reported by the CAP-certified laboratories for SurePath preparation $[20,21]$. The comparative reports were initially carried out in the period from August 2015 to March 2017, at 3 months intervals. After confirming the good quality of the cytopathological assessment, the intervals were extended to 12 months. The basic protocol also included the percentage rates estimation of HRHPV14-positive results within all cytologic diagnoses. For the cytologic-histologic correlations $(\mathrm{CHC})$, the CQI protocol was based on bi-directional scenarios published by the 2012 CAP gynecologic cytopathology consensus conference [22]. The performed review included the percentage of concordant and discrepant diagnoses, as well as the positive predictive value (PPV) of a positive cytology result, which is the preferred standard CHC metric for gynecologic cytopathology [22].

Tables 1 and 2 present the overview of correlating pairs used in the cytologicimmunocytochemical-histologic correlations (CICHC) assessment in our study. Table 1 shows positive $\mathrm{CHC}$, defined as correlations of abnormal cytology or positive $\mathrm{p} 16 / \mathrm{Ki} 67$ with positive histopathology (LSIL(CIN1)/HSIL(CIN2)/HSIL(CIN3)/ AIS or cervical cancer). Table 2 shows a negative $\mathrm{CICHC}$, defined as any abnormal cytology or positive/negative p16/Ki67 result that corresponds to a negative histopathological result and vice versa. Each diagnosis in the left column refers to each diagnosis in the right column in the Tables 1 and 2.

Table 1. Selected QA/QC tools used in CQI protocol-groups of positive cytologic-immunocytochemical-histologic correlations with modification for the study *.

\begin{tabular}{cc}
\hline LBC, P16/Ki67 Diagnosis & Postcolposcopy Biopsy Result \\
LSIL & LSIL (CIN1) \\
ASC-US HRHPV14+ve & HSIL (CIN2) \\
HSIL & HSIL (CIN3) \\
SIL & HSIL unspecified \\
AIS & AIS \\
CA & CA \\
p16/Ki67-positive & \\
\hline
\end{tabular}

${ }^{*}$ Each combination of cytology results in the left column with histology results in the right column is a positive correlation. Abbreviations: QA/QC, quality assessment and quality control; CQI, continuous quality improvement; LBC, liquid-based cytology; LSIL, p16/Ki67, p16/Ki67 dual staining test; low-grade squamous intraepithelial lesion; HSIL, high-grade squamous intraepithelial lesion; SIL, squamous intraepithelial lesion; AIS, adenocarcinoma in situ; CA, cancer of the cervix; LSIL (CIN1), histologic low-grade squamous intraepithelial lesion; HSIL (CIN2), histologic high-grade squamous intraepithelial lesion with a quantification of cervical intraepithelial neoplasia in grade 2; HSIL (CIN3), histologic high-grade squamous intraepithelial lesion with a quantification of cervical intraepithelial neoplasia in grade $3 ;+$ ve, -positive.

Table 2. Selected QA/QC tools used in CQI protocol-groups of negative cytologic-immunocytochemical-histologic correlations with modification for the study *.

\begin{tabular}{cc}
\hline LBC, P16/Ki67 Diagnosis & Postcolposcopy Biopsy Result \\
\hline LSIL & negative \\
ASC-US HRHPV14+ve & \\
HSIL & \\
SIL & \\
CA & \\
p16/Ki67-positive & \\
\hline
\end{tabular}


Table 2. Cont.

\begin{tabular}{cc}
\hline LBC, P16/Ki67 Diagnosis & Postcolposcopy Biopsy Result \\
\hline & LSIL (CIN1) \\
NILM & HSIL (CIN2) \\
p16/Ki67-negative & HSIL (CIN3) \\
& HSIL unspecified \\
& AIS \\
\hline
\end{tabular}

* Each combination of cytology results in the left column with histology results in the right column is a negative correlation. Abbreviations: $\mathrm{QA} / \mathrm{QC}$, quality assessment and quality control; CQI, continuous quality improvement; LBC, liquid-based cytology; LSIL, p16/Ki67, p16/Ki67 dual staining test; low-grade squamous intraepithelial lesion; HSIL, high-grade squamous intraepithelial lesion; SIL, squamous intraepithelial lesion; AIS, adenocarcinoma in situ; CA, cancer of the cervix; LSIL (CIN1), histologic low-grade squamous intraepithelial lesion; HSIL (CIN2), histologic high-grade squamous intraepithelial lesion with a quantification of cervical intraepithelial neoplasia in grade 2; HSIL (CIN3), histologic high-grade squamous intraepithelial lesion with a quantification of cervical intraepithelial neoplasia in grade $3 ;+$ ve, -positive.

\subsubsection{QA/QC Extended Protocol}

To maximize the diagnostic-therapeutic safety, in the initial period of the study, the enhanced safety protocol was used, including the CQI protocol extended with an additional quality control procedure for the LBC interpretation. After receiving the batch of LBC slides, a cytopathologist performed rapid prescreening (RPS) with the turret method, that is a quick (not exceeding one minute) morphological review [23]. During RPS, slides were classified into one of 3 groups: suspicious, negative, and unsatisfactory. At this stage, a cytopathologist did not use the clinical data and the RPS results were not used until the routine screening was completed. The routine screening was performed as usual [24]. The third stage of cytological evaluation was RPS, and determining the routine screening results' correlation with re-evaluation of all abnormal, suspicious, and unsatisfactory LBC. The final cytological diagnosis was established after the third stage was completed. RPS and re-evaluation of $100 \%$ abnormal LBC were performed after routine screening, using internal QA/QC tools in our ES protocol, which are recommended as the most effective for gynecologic cytopathology among various retrospective and prospective screening methods [23].

In both protocols (basic and extended), all LBC slides interpreted as unsatisfactory were prepared again at the request of the center, and the diagnostic procedure was repeated in its entirety.

\subsection{Colposcopic Biopsy and Histology}

The cases which were p16/Ki67-positive, ASC-US or LSIL HRHP14-positive, or with cytologic ASC-H or HSIL, regardless of HRHPV14 status, in accordance with the Polish recommendations with extension to the 2012 and 2015 ASCCP guidelines, were referred for a colposcopy with biopsy, performed by Center's gynecologists $[3,10,25,26]$. The minimal colposcopy protocol requirements included endocervical sampling, direct biopsies for all cases if abnormal colposcopic findings (or suspicious for invasion) were identified (based on 2011 IFCPC nomenclature), and random biopsies when no abnormal colposcopic findings were found and new-SCJ was visible. The histologic diagnoses of cervical biopsies and endocervical curettages with brushings were reviewed according to LAST 2012 and WHO/IARC 2014 terminology by a gynecological pathologist employed by the Center $[27,28]$. Colposcopies performed outside the Center, and/or histologic reports interpreted by someone other than Center pathologists, were not included in the study due to different colposcopy protocols and/or different histologic nomenclature and/or morphologic criteria used. The reevaluation of cytology, histology, and colposcopic findings was always performed when cyto-immunocytochemical-colposcopic-histologic discrepancies occurred, including ASC-H and HSIL in cytology with negative or LSIL (CIN1) biopsy results, and were made in direct or phone/online multidisciplinary team meetings. 


\section{Results}

\subsection{LBS Reporting Rates}

The age range of women included in the study was 15 to 92 years, with an average of 40.9 years, and the other group characteristics were: middle and higher economic status, mostly ( $>95 \%)$ from urbanized areas, and at least a secondary level of education. The total number of screening tests performed in the analyzed 5-year period was 30.066, including 20.605 cases of LBC reports, with 8331 HRHPV14 and 1130 p16/Ki67 tests available based on standardized indications $[10,14,25,26]$ or at the request of patients (as cotesting plus test). The number and type of test performed (LBC, HRHPV14, and p16/Ki67) in subsequent years are listed in Table 3. The number of tests performed in individual age groups is presented in Table 4. Most LBC, HRHPV14, and p16/Ki67 testing was performed in the 30-39 age group.

Table 3. Numbers of LBC, HRHPV14, and p16/Ki67 tests by year between August 2015 and July 2020.

\begin{tabular}{ccccc}
\hline Year & LBC, No. & HRHPV14, No. (\%) $\mathbf{1}^{\mathbf{1}}$ & p16/Ki67, No. (\%) $\mathbf{2}^{\mathbf{2}}$ & Total, No. \\
\hline 2015 & 2299 & $453(19.7)$ & $141(6.1)$ & 2893 \\
(August-December) & 5131 & $1206(23.5)$ & $192(3.7)$ & 6529 \\
2016 & 4475 & $1305(29.2)$ & $239(5.3)$ & 6019 \\
2017 & 3833 & $2056(53.6)$ & $241(6.3)$ & 6130 \\
2018 & 3411 & $2412(70.7)$ & $227(6.7)$ & 6050 \\
2019 & 1456 & $899(61.7)$ & $90(6.2)$ & 2445 \\
2020 (January-July) & 20,605 & $8331(40.4)$ & $1130(5.5)$ & 30,066 \\
Total & & & \\
\hline
\end{tabular}

Abbreviations: LBC, liquid-based cytology; HRHPV14, 14 high-risk types human papillomavirus test; p16/Ki67, p16/Ki67 dual staining test; ${ }^{1} \%$ LBC with HRHPV14 results; ${ }^{2} \%$ LBC results with p16/Ki67 results.

Table 4. Age-stratified LBS tests reporting rates.

\begin{tabular}{ccccc}
\hline Age, Years & LBC, No. & HRHPV14, No. & p16/Ki67, No. & Total, No. \\
\hline$<21$ & 226 & 40 & 16 & 282 \\
$21-24$ & 1224 & 373 & 98 & 1695 \\
$25-29$ & 2410 & 908 & 212 & 3530 \\
$30-39$ & 7285 & 3166 & 466 & 10,917 \\
$40-49$ & 4613 & 2248 & 219 & 7080 \\
$50-59$ & 2481 & 939 & 36 & 3486 \\
$60-65$ & 1384 & 424 & 17 & 1844 \\
$\geq 66$ & 982 & 233 & 1130 & 1232 \\
Total & 20,605 & 8331 & 30,066 \\
\hline
\end{tabular}

Abbreviations: LBS, liquid-based screening; LBC, liquid-based cytology; HRHPV14, 14 high-risk types human papillomavirus test; p16/Ki67, p16/Ki67 dual staining test.

In the analyzed population, the overall NILM, ASC-US, LSIL, ASC-H, HSIL, and AGC reporting rates were $93.9,3.4,2.0,0.22,0.24$, and $0.11 \%$, respectively. The percentage of HRHPV14 tests results was $37.7,82.8,87.6,84.8,87.8$, and $65.2 \%$, respectively, for the indicated above cytological diagnoses. The percentage of HRHPV14-positive tests results was $8.4,48.9,77.2,84.6,90.7$, and $26.7 \%$, respectively, for the corresponding cytological diagnoses. The percentage of $\mathrm{p} 16 / \mathrm{Ki} 67$ tests results for the indicated cytological diagnoses was $2.4,46.8,65.0,67.4,69.4$, and $34.8 \%$, respectively. The percentage of p16/Ki67-positive tests results for the indicated cytological diagnoses was 17.4, 30.0, 35.2, 87.1, 91.2, and $62.5 \%$, respectively (Table 5). 
Table 5. Overall NILM, ASC-US, LSIL, ASC-H, HSIL, and AGC reporting rates in the study group with relevant HRHPV14 and $\mathrm{p} 16 / \mathrm{Ki} 67$ results.

\begin{tabular}{|c|c|c|c|c|c|}
\hline $\begin{array}{l}\text { LBC Test } \\
\text { Result }\end{array}$ & Cases, No (\%) ${ }^{1}$ & $\begin{array}{c}\text { HRHPV14 Results, } \\
\text { No }(\%)^{2}\end{array}$ & $\begin{array}{c}\text { HRHPV14+ve } \\
\text { Results, No (\%) }\end{array}$ & $\begin{array}{c}\text { P16/Ki67 Results, } \\
\text { No }(\%)^{2}\end{array}$ & $\begin{array}{c}\text { P16/Ki67+ve } \\
\text { Results, No (\%) } 4\end{array}$ \\
\hline NILM & $19,338(93.9)$ & 7283 (37.7) & $612(8.4)$ & $458(2.4)$ & $80(17.4)$ \\
\hline ASC-US & $690(3.4)$ & $571(82.8)$ & $279(48.9)$ & $323(46.8)$ & $97(30.0)$ \\
\hline LSIL & $420(2.0)$ & $368(87.6)$ & $284(77.2)$ & $273(65.0)$ & $96(35.2)$ \\
\hline ASC-H & $46(0.22)$ & $39(84.8)$ & $33(84.6)$ & $31(67.4)$ & $27(87.1)$ \\
\hline HSIL & $49(0.24)$ & $43(87.8)$ & $39(90.7)$ & $34(69.4)$ & $31(91.2)$ \\
\hline AGC & $23(0.11)$ & $15(65.2)$ & $4(26.7)$ & $8(34.8)$ & $5(62.5)$ \\
\hline $\mathrm{ADC}$ & $2(0.01)$ & $1(50.0)$ & $0(0.0)$ & $1(50.0)$ & $1(100.0)$ \\
\hline SCC & $2(0.01)$ & $0(0.0)$ & $0(0.0)$ & $1(50.0)$ & $1(100.0)$ \\
\hline unsatisfactory & $35(0.17)$ & $11(31.4)$ & $5(45.5)$ & $1(2.9)$ & $1(100.0)$ \\
\hline Total & 20,605 & $8331(40.4)$ & $1256(15.1)$ & $1130(5.5)$ & $339(30.0)$ \\
\hline
\end{tabular}

Abbreviations: NILM, negative for intraepithelial lesion or malignancy; ASC-US, atypical squamous cells of undetermined significance; LSIL, low-grade squamous intraepithelial lesion; ASC-H, atypical squamous cells-cannot excluded HSIL; HSIL, high-grade squamous intraepithelial lesion; AGC, atypical glandular cells; ADC, adenocarcinoma; SCC, squamous cell carcinoma; LBC, liquid-based cytology; HRHPV14, 14 high-risk types human papillomavirus test; +ve, positive; p16/Ki67, p16/Ki67 dual staining test; ${ }^{1} \%$ of all LBC cases; ${ }^{2} \%$ of results defined in the first column; ${ }^{3} \%$ results defined in the first column with HRHPV14 results; ${ }^{4} \%$ results defined in the first column with p16/Ki67 results.

Age-stratified NILM, ASC-US, LSIL, ASC-H, HSIL, and AGC reporting rates were analyzed in eight age groups and all of the results are presented in Table 6. The NILM, ASC-US, LSIL, ASC-H, HSIL, and AGC reporting rates were highest in the group (age group No (\%)): 7 (96.6), $3(4.1), 1(6.6), 5(0.35), 7(0.36)$, and 5 (0.26), respectively.

Table 6. Age-stratified NILM, ASC-US, LSIL, ASC-H, HSIL, and AGC reporting rates in the study group.

\begin{tabular}{cccccccc}
\hline Group No & Age, Years & NILM, No (\%) & $\begin{array}{c}\text { ASC-US No } \\
(\%)\end{array}$ & LSIL No (\%) & $\begin{array}{c}\text { ASC-H No } \\
(\%)\end{array}$ & HSIL No (\%) & AGC No (\%) \\
\hline 1. & $<21$ & $204(90.3)$ & $6(2.7)$ & $15(6.6)$ & $0(0.0)$ & $0(0.0)$ & $0(0.0)$ \\
2. & $21-24$ & $1116(91.2)$ & $45(3.7)$ & $56(4.6)$ & $3(0.25)$ & $2(0.16)$ & $0(0.0)$ \\
3. & $25-29$ & $2194(91.0)$ & $99(4.1)$ & $91(3.8)$ & $7(0.29)$ & $8(0.33)$ & $3(0.13)$ \\
4. & $30-39$ & $6835(93.8)$ & $241(3.3)$ & $153(2.1)$ & $18(0.25)$ & $23(0.32)$ & $2(0.03)$ \\
5. & $40-49$ & $4333(94.0)$ & $168(3.6)$ & $71(1.5)$ & $16(0.35)$ & $9(0.20)$ & $12(0.26)$ \\
6. & $50-59$ & $2386(96.2)$ & $66(2.7)$ & $18(0.73)$ & $1(0.04)$ & $1(0.04)$ & $4(0.16)$ \\
7. & $60-65$ & $1337(96.6)$ & $30(2.2)$ & $8(0.58)$ & $1(0.07)$ & $5(0.36)$ & $1(0.07)$ \\
8. & $\geq 66$ & $933(95.0)$ & $35(3.6)$ & $8(0.82)$ & $0(0.0)$ & $1(0.10)$ & $1(0.10)$ \\
& Total & $19,338(93.9)$ & $690(3.4)$ & $420(2.0)$ & $46(0.22)$ & $49(0.24)$ & $23(0.11)$ \\
\hline
\end{tabular}

Abbreviations: NILM, negative for intraepithelial lesion or malignancy; ASC-US, atypical squamous cells of undetermined significance; LSIL, low-grade squamous intraepithelial lesion; ASC-H, atypical squamous cells-cannot excluded HSIL; HSIL, high-grade squamous intraepithelial lesion; AGC, atypical glandular cells.

\subsection{Histologic Correlation for LBS Results}

The final analysis included cases with a performed colposcopy with biopsy within 3 months of obtaining the initial screening tests results. The cyto-histologic correlation results are summarized in Table 7 . Of 20.605 cases, 415 ( $2.0 \%$ of all study groups) with LBC reports had a colposcopy with a biopsy procedure in our Center, from a total of 846 women referred for a colposcopy. A total of 93 cases of HSIL (CIN2+) were diagnosed, which was $22.4 \%$ of the total with biopsy results. No cases of SCC or AIS were identified. The highest number of HSIL (CIN2+) diagnoses (23 cases) were found among ASC-US cytology diagnoses, which was $5.5 \%$ of the total biopsy results. In other cytological diagnoses, it was reported as follows (No of detected HSIL (CIN2+) cases; \% of total biopsy results): for NILM (17; 4.1\%), LSIL (21; 5.1\%), ASC-H (11;2.7\%), HSIL (20; $4.8 \%)$, AGC $(1 ; 0.24 \%)$, SCC/AIS $(0.0 \%)$. The following histological diagnoses were not included in the final analysis: B-DLBCL NOS (1 case), CGIN (1 case), SIL ( 3 cases), and HG CGIN (1 case). 
Table 7. The histologic correlation for LBC (CHC).

\begin{tabular}{|c|c|c|c|c|c|}
\hline \multirow[b]{2}{*}{ LBC Result } & \multirow{2}{*}{$\begin{array}{c}\text { No }\left(\%^{1} / \%^{2}\right) \\
\text { Total Biopsy Results }\end{array}$} & \multicolumn{4}{|c|}{ Histology Result, No (\%) ${ }^{3}$} \\
\hline & & Negative & LSIL (CIN1) & HSIL (CIN2) & HSIL (CIN3+) \\
\hline NILM & $62(0.32 / 14.9)$ & $37(59.7)$ & $8(12.9)$ & $12(19.4)$ & $5(8.1)$ \\
\hline ASC-US & $153(22.2 / 36.9)$ & $83(54.3)$ & $47(30.7)$ & $14(9.2)$ & $9(5.9)$ \\
\hline LSIL & $139(33.1 / 33.5)$ & $47(33.8)$ & $71(51.1)$ & $16(11.5)$ & $5(3.6)$ \\
\hline ASC-H & $25(54.4 / 6.0)$ & $8(32.0)$ & $6(24.0)$ & $5(20.0)$ & $6(24.0)$ \\
\hline HSIL & $26(53.1 / 6.3)$ & $4(15.4)$ & $2(7.7)$ & $6(23.1)$ & $14(53.9)$ \\
\hline AGC & $10(43.5 / 2.4)$ & $9(90.0)$ & $0(0.0)$ & $0(0.0)$ & $1(10.0)$ \\
\hline SCC/AIS & $0(0.0 / 0.0)$ & $0(0.0)$ & $0(0.0)$ & $0(0.0)$ & $0(0.0)$ \\
\hline Total & $415(2.0 / 100.0)$ & $188(45.3)$ & $134(32.3)$ & $53(12.8)$ & $40(9.6)$ \\
\hline
\end{tabular}

Abbreviations: LBC, liquid-based cytology; CHC, cyto-histologic correlations; NILM, negative for intraepithelial lesion or malignancy; ASC-US, atypical squamous cells of undetermined significance; LSIL, low-grade squamous intraepithelial lesion; ASC-H, atypical squamous cells-cannot excluded HSIL; HSIL, high-grade squamous intraepithelial lesion; AGC, atypical glandular cells; SCC, squamous cell carcinoma; AIS, adenocarcinoma in situ; LSIL (CIN1), histologic low-grade squamous intraepithelial lesion; HSIL (CIN2), histologic high-grade squamous intraepithelial lesion with a quantification of cervical intraepithelial neoplasia in grade 2; HSIL (CIN3+), histologic high-grade squamous intraepithelial lesion with a quantification of cervical intraepithelial neoplasia in grade 3 or worse; ${ }^{1} \%$ of results defined in the first column; ${ }^{2} \%$ of all biopsy results; ${ }^{3} \%$ of total biopsy results for the LBC result defined in the first column.

The cyto-virologic-histologic correlations (CVHC) results are summarized in Table 8. Of 20.605 cases, 402 (2.0\% of all study group) with LBC and HRHPV14 results had a colposcopy with a biopsy procedure in our Center. Negative and LSIL (CIN1) histology results were found in $76.4 \%$ of all HRHPV14-positive cases and in $92.0 \%$ of HRHPV14negative cases. The positive histology results (HSIL (CIN2+)) in $97.8 \%$ (91 cases) were HRHPV14-positive, and in 2.2\% (2 cases), HRHPV14-negative (both HSIL in cytology). In the study group, 17 cases of HSIL (CIN2+) NILM HRHPV14-positive were found, which was $18.7 \%$ of all HSIL (CIN2+) diagnoses.

Table 8. The histologic correlation for LBC and HRHPV14 results (CVHC).

\begin{tabular}{|c|c|c|c|c|c|}
\hline \multirow[b]{2}{*}{ LBC Result } & \multicolumn{2}{|l|}{ No $(\%)^{1}$} & \multicolumn{3}{|c|}{ Histology Result, No (\%) ${ }^{2}$} \\
\hline & TBR with HPV Results & Negative & LSIL (CIN1) & HSIL (CIN2) & HSIL (CIN3+) \\
\hline NILM & $61(8.4)$ & $36(59.0)$ & $8(13.1)$ & $12(19.7)$ & $5(8.2)$ \\
\hline HRHPV14+ve & $60(8.2)$ & $35(58.3)$ & $8(13.3)$ & $12(20.0)$ & $5(8.3)$ \\
\hline HRHPV14-ve & $1(0.01)$ & $1(100.0)$ & $0(0.0)$ & $0(0.0)$ & $0(0.0)$ \\
\hline ASC-US & $147(25.7)$ & $82(55.8)$ & $42(28.6)$ & $14(9.5)$ & $9(6.1)$ \\
\hline HRHPV14+ve & $139(24.3)$ & $75(54.0)$ & $41(29.5)$ & $14(10.1)$ & $9(6.5)$ \\
\hline HRHPV14-ve & $8(1.4)$ & $7(87.5)$ & $1(12.5)$ & $0(0.0)$ & $0(0.0)$ \\
\hline LSIL & $136(37.0)$ & $45(33.1)$ & $71(52.2)$ & $15(11.0)$ & $5(3.7)$ \\
\hline HRHPV14+ve & $131(35.6)$ & $42(32.1)$ & $69(52.7)$ & $15(11.5)$ & $5(3.8)$ \\
\hline HRHPV14-ve & $5(1.4)$ & $3(60.0)$ & $2(40.0)$ & $0(0.0)$ & $0(0.0)$ \\
\hline ASC-H & $25(64.1)$ & $8(32.0)$ & $6(24.0)$ & $5(20.0)$ & $6(24.0)$ \\
\hline HRHPV14+ve & $22(56.4)$ & $6(27.3)$ & $5(22.7)$ & $5(22.7)$ & $6(27.3)$ \\
\hline HRHPV14-ve & $3(7.7)$ & $2(66.7)$ & $1(33.3)$ & $0(0.0)$ & $0(0.0)$ \\
\hline HSIL & $25(58.1)$ & $4(16.0)$ & $2(8.0)$ & $6(24.0)$ & $13(52.0)$ \\
\hline HRHPV14+ve & $23(53.5)$ & $4(17.4)$ & $2(8.7)$ & $5(21.7)$ & 12 (52.2) \\
\hline HRHPV14-ve & $2(4.7)$ & $0(0.0)$ & $0(0.0)$ & $1(50.0)$ & $1(50.0)$ \\
\hline AGC & $8(53.3)$ & 7 (87.5) & $0(0.0)$ & $0(0.0)$ & $1(12.5)$ \\
\hline HRHPV14+ve & $2(13.3)$ & $1(50.0)$ & $0(0.0)$ & $0(0.0)$ & $1(50.0)$ \\
\hline HRHPV14-ve & $6(40.0)$ & $6(100.0)$ & $0(0.0)$ & $0(0.0)$ & $0(0.0)$ \\
\hline Total & $402(4.8)$ & $182(45.3)$ & $129(32.1)$ & $52(12.9)$ & $39(9.7)$ \\
\hline HRHPV14+ve & $377(4.5)$ & $163(43.2)$ & $125(33.2)$ & $51(13.5)$ & $38(10.1)$ \\
\hline HRHPV14-ve & $25(0.30)$ & $19(76.0)$ & $4(16.0)$ & $1(4.0)$ & $1(40)$ \\
\hline
\end{tabular}

Abbreviations: LBC, liquid-based cytology; HRHPV14, 14 high-risk types human papillomavirus test; +ve, positive; -ve, negative; CVHC, cyto-viro-histologic correlations; TBR, total biopsy results; NILM, negative for intraepithelial lesion or malignancy; ASC-US, atypical squamous cells of undetermined significance; LSIL, low-grade squamous intraepithelial lesion; ASC-H, atypical squamous cells-cannot excluded HSIL; HSIL, high-grade squamous intraepithelial lesion; AGC, atypical glandular cells; LSIL (CIN1), histologic low-grade squamous intraepithelial lesion; HSIL (CIN2), histologic high-grade squamous intraepithelial lesion with a quantification of cervical intraepithelial neoplasia in grade 2; HSIL (CIN3+), histologic high-grade squamous intraepithelial lesion with a quantification of cervical intraepithelial neoplasia in grade 3 or worse; ${ }^{1} \%$ of results defined in the first column; ${ }^{2} \%$ of total biopsy results for the LBC result and HRHPV14 status defined in the first column. 
The cyto-virologic-immunocytochemical-histologic correlations (CVICHC) results are summarized in Table 9. Of 20.605 cases, 375 (1.8\% of all study group) with LBC, HRHPV14 and p16/Ki67 test results had a colposcopy with a biopsy procedure in our Center. Negative and LSIL (CIN1) histology results were found in 75.9\% of all HRHPV14positive cases and in $91.3 \%$ of HRHPV14-negative cases. The positive histology results (HSIL (CIN2+)) in $94.1 \%$ (80 cases) tested p16/Ki67-positive, and in $5.9 \%$ (5 cases) were p16/Ki67-negative in the HRHPV14-positive group. In the study group, 2 HSIL (CIN2+) cases which were p16/Ki67-positive and HRHPV14-negative were found, which was $100 \%$ of all HSIL (CIN2+) HRHPV14-negative results.

Table 9. The histologic correlation for LBC, HRHPV14, and p16/Ki67 results (CVICHC).

\begin{tabular}{|c|c|c|c|c|c|}
\hline \multirow[b]{2}{*}{ LBC Result } & \multirow{2}{*}{$\begin{array}{c}\text { No (\%) } \\
\text { TBR with HRHPV14 } \\
+ \text { P16/Ki67 Results }\end{array}$} & \multicolumn{4}{|c|}{ Histology Result, No (\%) ${ }^{2}$} \\
\hline & & Negative & LSIL (CIN1) & HSIL (CIN2) & HSIL (CIN3+) \\
\hline NILM & $59(12.9)$ & $34(57.6)$ & $8(13.6)$ & $12(20.3)$ & $5(8.5)$ \\
\hline HPV+ve & $58(12.7)$ & $33(56.9)$ & $8(13.8)$ & $12(20.7)$ & $5(8.6)$ \\
\hline p16/Ki67 (+) & 47 (10.3) & $23(48.9)$ & 7 (14.9) & $12(25.5)$ & $5(10.6)$ \\
\hline p16/Ki67 (-) & $11(2.4)$ & $10(90.9)$ & $1(9.1)$ & $0(0.0)$ & $0(0.0)$ \\
\hline HPV-ve & $1(0.22)$ & $1(100.0)$ & $0(0.0)$ & $0(0.0)$ & $0(0.0)$ \\
\hline p16/Ki67 (+) & $1(0.22)$ & $1(100.0)$ & $0(0.0)$ & $0(0.0)$ & $0(0.0)$ \\
\hline p16/Ki67 (-) & $0(0.0)$ & $0(0.0)$ & $0(0.0)$ & $0(0.0)$ & $0(0.0)$ \\
\hline ASC-US/LSIL & $268(45.0)$ & $118(44.0)$ & $107(39.9)$ & $29(10.8)$ & $14(5.2)$ \\
\hline HPV+ve & $252(42.3)$ & $107(42.5)$ & $102(40.5)$ & $29(11.5)$ & $14(5.6)$ \\
\hline p16/Ki67 (+) & $126(21.1)$ & $30(23.8)$ & $55(43.7)$ & $27(21.4)$ & $14(11.1)$ \\
\hline p16/Ki67 (-) & $126(21.1)$ & $77(61.1)$ & $47(37.3)$ & $2(1.6)$ & $0(0.0)$ \\
\hline HPV-ve & $16(2.7)$ & $11(68.8)$ & $5(31.3)$ & $0(0.0)$ & $0(0.0)$ \\
\hline p16/Ki67 (+) & $12(2.0)$ & $8(66.7)$ & $4(33.3)$ & $0(0.0)$ & $0(0.0)$ \\
\hline p16/Ki67 (-) & $4(0.67)$ & $3(75.0)$ & $1(25.0)$ & $0(0.0)$ & $0(0.0)$ \\
\hline ASC-H+ & $48(65.8)$ & $14(29.2)$ & $7(14.6)$ & $10(20.8)$ & $17(35.4)$ \\
\hline HPV+ve & $42(57.5)$ & $11(26.2)$ & $6(14.3)$ & $9(21.4)$ & $16(38.1)$ \\
\hline p16/Ki67 (+) & $35(48.0)$ & $9(25.7)$ & $4(11.4)$ & $7(20.0)$ & $15(42.9)$ \\
\hline p16/Ki67 (-) & $7(9.6)$ & $2(28.6)$ & $2(28.6)$ & $2(28.6)$ & $1(14.3)$ \\
\hline HPV-ve & $6(8.2)$ & $3(50.0)$ & $1(16.7)$ & $1(16.7)$ & $1(16.7)$ \\
\hline p16/Ki67 (+) & $4(5.5)$ & $1(25.0)$ & $1(25.0)$ & $1(25.0)$ & $1(25.0)$ \\
\hline p16/Ki67 (-) & $2(2.7)$ & $2(100.0)$ & $0(0.0)$ & $0(0.0)$ & $0(0.0)$ \\
\hline Total & $375(33.3)$ & $166(44.3)$ & $122(32.5)$ & $51(13.6)$ & $36(9.6)$ \\
\hline HPV+ve & $352(31, .2)$ & $151(42.9)$ & $116(33.0)$ & $50(14.2)$ & $35(9.9)$ \\
\hline p16/Ki67 (+) & $208(18.5)$ & $62(29.8)$ & $66(31.7)$ & $46(22.1)$ & $34(16.3)$ \\
\hline p16/Ki67 (-) & 144 (12.8) & $89(61.8)$ & $50(34.7)$ & $4(2.8)$ & $1(0.69)$ \\
\hline HPV-ve & $23(2.0)$ & $15(65.2)$ & $6(26.1)$ & $1(4.4)$ & $1(4.4)$ \\
\hline p16/Ki67 (+) & $17(1.5)$ & $10(58.8)$ & $5(29.4)$ & $1(5.9)$ & $1(5.9)$ \\
\hline p16/Ki67 (-) & $6(0.53)$ & $5(83.3)$ & $1(16.7)$ & $0(0.0)$ & $0(0.0)$ \\
\hline
\end{tabular}

Abbreviations: LBC, liquid-based cytology; HRHPV14, 14 high-risk types human papillomavirus test; p16/Ki67, p16/Ki67 dual staining test; +ve, positive; -ve, negative; CVICHC, cyto-virologic-immunocytochemical-histologic correlations; TBR, total biopsy results; NILM, negative for intraepithelial lesion or malignancy; ASC-US, atypical squamous cells of undetermined significance; LSIL, low-grade squamous intraepithelial lesion; ASC-H+, cytology results: ASC-H (atypical squamous cells-cannot excluded HSIL), HSIL (high-grade squamous intraepithelial lesion) or AGC (atypical glandular cells); LSIL (CIN1), histologic low-grade squamous intraepithelial lesion; HSIL (CIN2), histologic high-grade squamous intraepithelial lesion with a quantification of cervical intraepithelial neoplasia in grade 2; HSIL (CIN3+), histologic high-grade squamous intraepithelial lesion with a quantification of cervical intraepithelial neoplasia in grade 3 or worse; $1 \%$ of results defined in the first column; ${ }^{2} \%$ of total biopsy results for the LBC result, HRHPV14 and p16/Ki67 status defined in the first column.

\subsection{Basic and Extended QA/QC Protocols Results}

The CQI protocol was used throughout the study and remained the basic QC in the Center protocol at the end of the study. Its full application with PPV evaluation for cytology results was possible from the 4th QC round. The ES protocol was used in the study until July 2017 for QC Round 6. The confirmation of the statistical compliance of both protocols in the subsequent QC rounds (1-6) allowed for a departure from the labor-intensive and time-consuming additional procedure without losing the quality of the cytopathological assessment. In total, $9 \mathrm{QC}$ rounds were performed during our study. The first 5 rounds 
were performed, on average, every 3 months. Rounds 6 to 9 were performed in periods not exceeding 12 months. The next rounds, together with the increasing number of LBC cases assessed, are listed in Table 10.

Table 10. Following $Q C$ rounds with cumulative number of $L B C$ cases.

\begin{tabular}{ccc}
\hline QC Round, No & QC Date & No of LBC Cases, Cumulative \\
\hline 1 & September 2015 & 661 \\
2 & December 2015 & 2137 \\
3 & February 2016 & 3017 \\
4 & June 2016 & 6716 \\
5 & October 2016 & 8322 \\
6 & July 2017 & 12,293 \\
7 & July 2018 & 17,167 \\
8 & July 2019 & 20,605 \\
\hline
\end{tabular}

Abbreviations: QC, quality control; LBC, liquid-based cytology.

Figure 1 shows the cumulative percentage of ASC-US diagnoses in relation to all LBC diagnoses (upper curve) in the subsequent $\mathrm{QA} / \mathrm{QC}$ rounds, the cumulative percentage of HRHPV14-positive ASC-US diagnoses in relation to all LBC diagnoses (middle curve), and the cumulative percentage of HRHPV14-positive ASC-US diagnoses (presented as a decimal) over all ASC-US diagnoses (lower curve), and the corresponding trend curves. The lowest cumulative percentage of ASC-US diagnoses was $2.66 \%$ and the highest was $3.48 \%$, and all values were between the 10 th and 50 th percentile reported by CAP-accredited laboratories for SurePath LBC preparation. The cumulative percentage of HRHPV-positive ASC-US for the entire study was $1.64 \%$ of all the LBC diagnoses, and for ASC-US diagnoses it was $48.86 \%$.

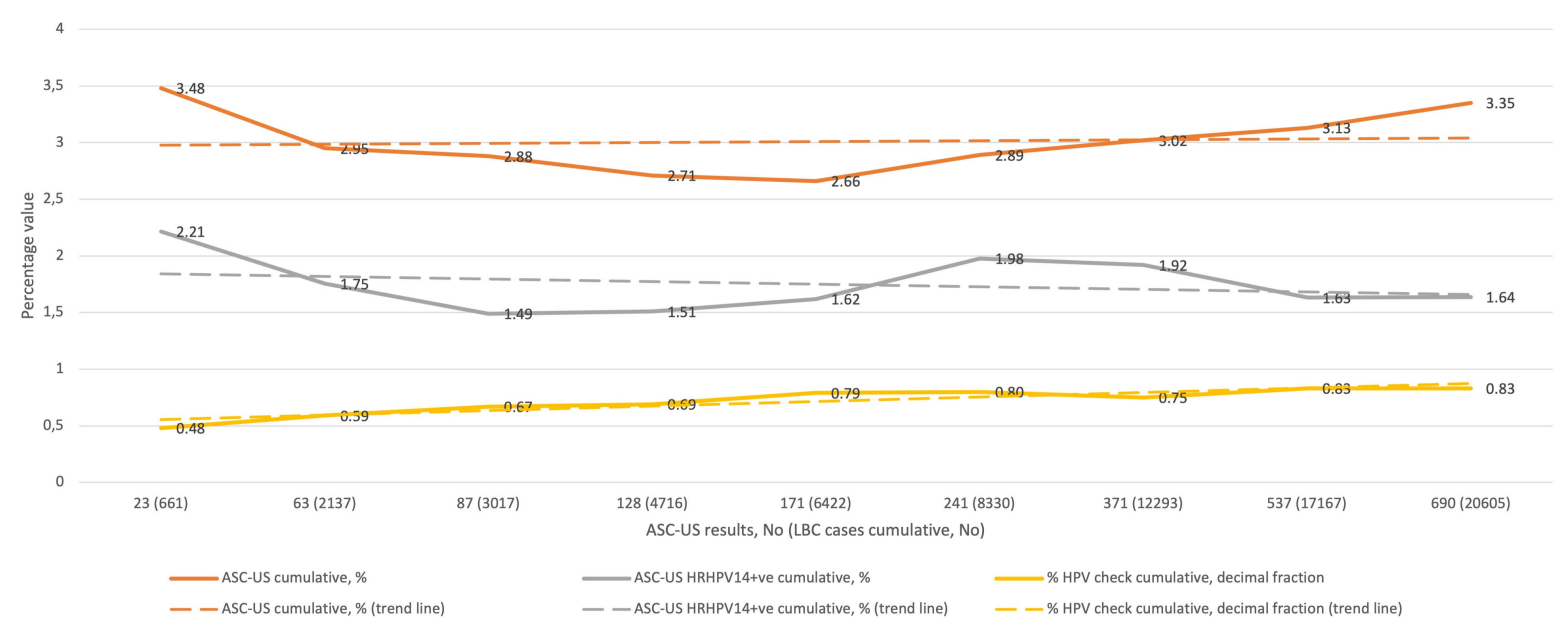

Figure 1. QC results for ASC-US in the study period (1-9 rounds). Abbreviations: QC, quality control; ASC-US, atypical squamous cells of undetermined significance; LBC, liquid-based cytology; HRHPV14, 14 high-risk types of human papillomavirus test; +ve, positive; HPV, HRHPV14.

Figure 2 shows the cumulative percentage of LSIL diagnoses in relation to all LBC diagnoses in the subsequent $\mathrm{QA} / \mathrm{QC}$ rounds (upper curve), the cumulative percentage of HRHPV14-positive LSIL diagnoses in relation to all LBC diagnoses (middle curve), and the cumulative percentage of HRHPV14-positive LSIL diagnoses (presented as a decimal) relative to all LSIL diagnoses (lower curve), and the corresponding trend curves. The lowest cumulative percentage of LSIL diagnoses was $1.51 \%$ and the highest was $2.04 \%$, and all values were between the 25th and 50th percentile reported for the SurePath LBC preparation. The cumulative percentage of HRHPV-positive LSIL for the entire study was $1.57 \%$ of all LBC diagnoses, and for LSIL diagnoses it was $77.17 \%$. 


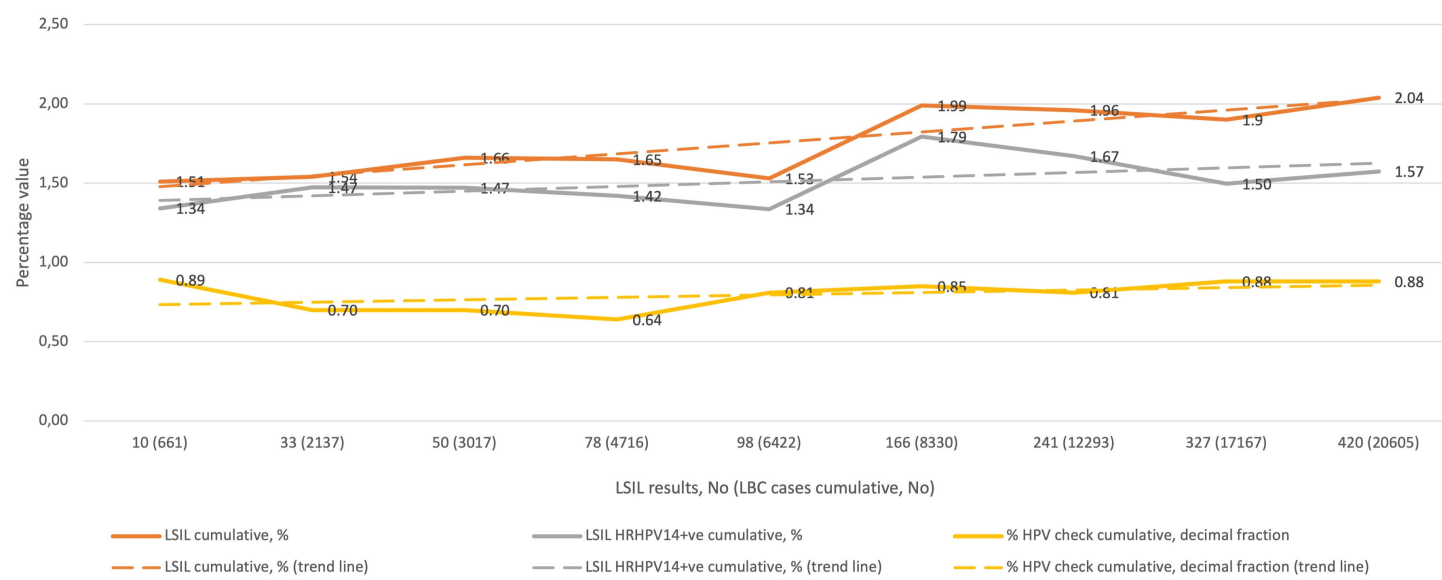

Figure 2. QC results for LSIL in the study period (1-9 rounds). Abbreviations: QC, quality control; LSIL, low-grade squamous intraepithelial lesion; LBC, liquid-based cytology; HRHPV14, 14 high-risk types human papillomavirus test; +ve; positive; HPV, HRHPV14.

Figure 3 shows the cumulative ratio of ASC to SIL diagnoses, and the corresponding trend curve in the subsequent QA/QC rounds. The lowest ASC/SIL ratio was 1.38 and the highest was 2.4, and all values were between the 25th and 75th percentile (most below 1.8, i.e., between the 25th and 50th percentile) reported for the SurePath LBC preparation. The cumulative ASC/SIL ratio for the entire study was 1.57 .

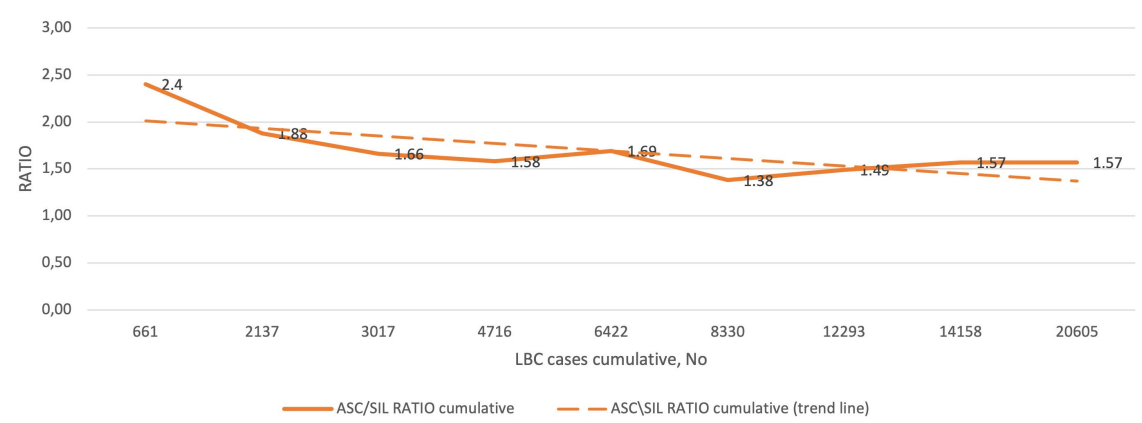

Figure 3. QC results for ASC/SIL in the study group (1-9 rounds). Abbreviations: QC, quality control; ASC, atypical squamous cells; SIL, squamous intraepithelial lesion; LBC, liquid-based cytology.

A summary of the complete results of the last QC round of the study is presented in Table 11. The PPV for positive (abnormal) LBC results in our study achieved $69.46 \%$.

Table 11. Cyto-virologic correlations (CVC) for the study population for the CQI protocol (round 9).

\begin{tabular}{|c|c|c|c|}
\hline LBC Result & No $(\%)^{1}$ & HRHPV14 Results, No (\%) ${ }^{2}$ & HRHPV14+ve, No (\%) ${ }^{3}$ \\
\hline NILM & 19,338 (93.85) & $7283(37.66)$ & $612(8.40)$ \\
\hline ASC-US & $690(3.35)$ & $571(82.75)$ & $279(48.86)$ \\
\hline ASC-H & $46(0.22)$ & $39(84.78)$ & 33 (84.62) \\
\hline LSIL & $420(2.04)$ & $368(87.62)$ & $284(77.17)$ \\
\hline HSIL & $49(0.24)$ & $43(87.76)$ & $39(90.70)$ \\
\hline ASC/SIL & 1.57 & $\mathrm{x}$ & $\mathrm{x}$ \\
\hline AGC & $23(0.11)$ & $15(65.22)$ & $4(26.67)$ \\
\hline unsatisfactory & $35(0.17)$ & $11(31.43)$ & $5(45.45)$ \\
\hline SCC & $2(0.01)$ & $0(0.00)$ & $0(0.00)$ \\
\hline ADC & $2(0.01)$ & $1(50.00)$ & $0(0.00)$ \\
\hline Total & $20,605(100.00)$ & $8331(40.43)$ & $1256(15.08)$ \\
\hline
\end{tabular}

Abbreviations: CVC, cyto-virologic correlations; CQI, continuous quality improvement; LBC, liquid-based cytology; HRHPV14, 14 high-risk types human papillomavirus test; +ve, positive; NILM, negative for intraepithelial lesion or malignancy; ASC-US, atypical squamous cells of undetermined significance; ASC-H, atypical squamous cells-cannot excluded HSIL; LSIL, low-grade squamous intraepithelial lesion; HSIL, high-grade squamous intraepithelial lesion; ASC/SIL, atypical squamous cells/squamous intraepithelial lesion ratio; AGC, atypical glandular cells; SCC, squamous cell carcinoma; ADC, adenocarcinoma; $1 \%$ of total LBC results; ${ }^{2} \%$ of results defined in the first column; $3 \%$ of results in the third column. 


\section{Discussion}

In this aged-stratified study, data from private-based, opportunistic cervical cancer screening, including LBC, HRHPV14, and p16/Ki67 dual staining tests results, and their reporting rates with histologic correlations, were analyzed. Our investigation covered a total of 30.066 LBS screening tests assessed in a 5-year period in a Polish urban area population, and were highly consistent with other data, including ASCCP-sponsored US studies, data from non-US laboratories accredited by CAP, and with available data from Western European countries. Our experience with combinations of screening tests results provides the baseline data in cervical cancer screening in Poland, and when considering p16/Ki67 test results analysis, it also complements international data. Indirectly, this study demonstrates the applicability of the 2019 ASCCP risk-based guidelines for abnormal cervical cancer screening test results and cancer precursors outside the US. In the conditions of an ineffective public funds-based organized cervical cancer prevention program, privatebased screening can effectively carry out screening, significantly supporting the national public system.

The HRHPV prevalence in women with normal cytology ranges from $6.6 \%$ to $22.9 \%$, depending on geographic location. The estimated worldwide HPV DNA average rate is $10 \%$. In our HPV study, the prevalence of HRHPV14 in women with NILM cytology results was $8.4 \%$ and is the closest to the levels reported by Northern European countries [29,30]. In the analysis of our results, we included American, Asian, and European population data as the comparators. The similarities to the HRHPV14-positivity of our results in all categories of abnormal cytological diagnoses were noted. Our HRHPV14-positivity rate of $48.9 \%$ for ASC-US cytology cases was within the levels reported for the US conditions, including CAP (47.73\%) [31] and the Kaiser Permanente Northern California Foundation (48.39\%) [17]. A meta-analysis of Arbyn et al. showed an average HRHPV-positivity rate for ASC-US cytology of $43.0 \%$, but it should be remembered that slightly lower levels compared to ours in this case might be associated with the use of a different HRHPV test, which detects less high-risk types [32] than in our study. Meanwhile, the data from large-scale research carried out by CAP-accredited Chinese laboratories reports $48.7-54.7 \%$ for ASC-US HRHPV-positive cases $[33,34]$. Our results also demonstrated similar positive HRHPV rates in women with LSIL $(77.2 \%)$ as reported by American $(74.4-85.3 \%)$ [35,36], other CAP-accredited laboratories outside the US (75.2-79.2\%) [34,37], and Western European countries (76.0\%) [32]. Similarities in HRHPV-positivity rates for major cervical screening abnormalities encompassing ASC-H, HSIL, and AGC ( $84.6 \% ; 90.7 \%$ and $26.7 \%$, respectively) also were noted-for American reports and other CAP-accredited laboratories outside the US (78.07\%; 88.1-94.78\% and 16.47-27.78\%, respectively) [36,38]. An exception is the HRHPV-positive LSIL reporting rate, with higher levels (85.3\%) compared to our results $(77.2 \%)$ [35]. There are several potential reasons for the differences, such as the wider age range in our study, including low HPV prevalent age groups, the heterogeneity of patients, and/or the different HPV test used.

The reliable comparison of cytology and HRHPV-positivity reporting rates, along with the histology detection rates with some large randomized Western European studies, was impossible due to the use of conventional cytological preparation, different cytologic classifications, combining cytological diagnoses into groups, or due to narrower age ranges analyzed when compared to our study. The reliable comparison between our study, in terms of HRHPV-positivity and cytology results, with European data from the study of the New Technologies for Cervical Cancer screening (NTCC) Working Group (published in Lancet Oncology [39]), where HRHPV-positivity in 9.56\% of NILM was found, $45.95 \%$ of ASC-US, $72.05 \%$ of LSIL, and $92.11 \%$ of HSIL cytology, shows results all reporting rates similar to our own results.

The results of our study were highly consistent with large scale, ASCCP-sponsored investigations aimed at giving preliminary risk estimates for future guidelines. This high compliance rate has a particular relevance for the Polish interim guidelines for cervical cancer screening published in March 2021, as one of recommended screening approach is 
management according to the 2019 ASCCP-risk based guidelines. It might be possible that the introduction of the 2019 ASCCP guidelines in other Central-Eastern European countries with economic and cultural environments similar to that of Poland with conditions for proper QA/QC for gynecologic cytopathology.

The second goal of our investigation was to compare the obtained cytologic reporting rates with the data reported by CAP for the evaluation of gynecological cytopathology in percentile distributions. The analysis showed that our study results are within the ranges reported by the CAP-accredited laboratories, which confirms the possibility of effective application of the CAP cytopathology benchmarks in other geographic locations, and might substantiate the applicability of the 2019 ASCCP risk-based guidelines in Polish conditions. For the comparative analysis of cervical cytology reporting rates, we used the available benchmarking data reported by laboratories participating in the CAP accreditation program, after also implementing of the Bethesda 2014 system [21]. The distribution reporting rates for our LBC results were between the 25 th and 50th percentiles for the vast majority cytologic categories. The percentages for our cytological diagnoses of ASC-US, LSIL, ASC-H, HSIL, and AGC were 3.35\%, 2.04\%, 0.22\%, $0.24 \%$, and $0.11 \%$, respectively. The reporting rate in the percentile distribution for unsatisfactory cytology results (mostly too low cellularity) was $0.17 \%$, and also was between the 25 th and 50 th percentile, as well as the ASC-SIL ratio, which was 1.56. The analysis of our cytopathological evaluation trends clearly suggests that the increase in the number of abnormal cytological diagnoses of ASC-US and LSIL is associated with the increasing percentage of cotesting results in the study group, and thus with the growing share of informed cytology evaluation in all HRHPV-positive cases, which is consistent with the important conclusions of NTCC group from their randomized controlled trial [40]. The PPV of the abnormal LBC results (ASC-US+) in our study was $69.46 \%$, slightly below the range achieved by American laboratories (71-94\%) [22]. However, it should be remembered that these ranges come from the work published in 1996, when surveillance management was recommended for patients with ASC-US and indications for a colposcopy were different without the HRHPV status analysis. In addition, colposcopy protocols at that time allowed for biopsy much less frequently, hence the availability of histology reports was lower, and thus potentially the number of false positives was also lower. Independently, the heterogeneity of the studied groups may be a factor determining the PPV level. Comparative data for the Polish population in that matter are lacking.

The histopathologic examination results demonstrated that a substantial number of our patients (28.3\%) with NILM cytology HRHPV14-positive findings had HSIL (CIN2+), including $20.0 \%$ that were HSIL (CIN2). The histopathological results presented in other studies in the group of NILM HRHPV-positive patients differ significantly from $5.4 \%$ to $25.24 \%$, with our histopathologic reporting rates similar to the ASCCP studies. Among the NILM HPV-negative cases, we found no HSIL (CIN2+) cases. The findings of our study support the HPV-based strategy (primary HPV or cotesting) in the Polish screening model and/or the need of a further triage option. In HRHPV14-positive cases with abnormal LBC diagnoses, we also did not find differences in the histopathological results compared to CAP certified laboratories outside the US, with immediate referrals for a colposcopy with a biopsy conducted.

Incorporating p16/Ki67 dual-stained cytology in our study provides Polish data on the proposed new tool for HSIL-risk detection, registered by the FDA as an alternative to cytology-based triage in HPV-based models [15,41]. The p16/Ki67 triage (i.e., in HRHPVpositive women with cytological NILM who had cotesting) increases the effectiveness of HSIL detection, which was also confirmed by our study-10.3\% women in this group were p16/Ki67-positive, which allowed the detection of an additional 17 cases of HSIL (CIN2+), $19.54 \%$ of all detected HSIL (CIN2+). A total of $1130 \mathrm{p} 16 / \mathrm{Ki} 67$ tests were performed, of which $30 \%$ were positive, regardless of the age group and other screening tests results. Among the abnormal cytological diagnoses, the following percentages of $\mathrm{p} 16 / \mathrm{Ki} 67$-positive cases was found: $30.0 \%$ for ASC-US; 35.2\% for LSIL; $87.1 \%$ for ASC-H; $91.2 \%$ for HSIL; 
and $62.5 \%$ for AGC, which correlated with previous publications [42-44]. Similarities with other reports $[13,43]$ were also observed in virologic-immunocytochemical-histologic correlations. Among the HRHPV-positive, p16/Ki67-positive with negative biopsy results, $39.6 \%$ of p16/Ki67-positive cases were found; with histologic LSIL (CIN1), 54.3\%; and with histologic HSIL (CIN2 and CIN3), 90.1\%. It should be pointed, that evaluation of p16/Ki67 dual-staining diagnostic value is the subject of another study of our group.

This analysis was also performed to create a baseline for the formation of an appropriate management method in cervical cancer screening for the Polish population, and to achieve the fullest data possible on the Polish private-based secondary cervical cancer prevention, which is characterized by a high market share of private medical services. Most Polish women declared participation in private opportunistic cervical cancer screening [45], whilst population coverage by the population-based national screening system is very low $[6,7]$. This situation is far from the screening effectiveness requirements, which clearly indicates the need for urgent changes. The European guidelines indicate an advantage of the population-based organized screening over the opportunistic one. However, a well-designed population-based program may not achieve the effectiveness parameters if it does not include local conditions [4,7]-one size does not fit all. Women's preferences should be taken into account in the building of an effective model of secondary cervical cancer prevention in Poland and other countries with a similar history, screening tradition, and share of the private sector. Currently, as part of publicly funded care, the cytologybased opportunistic model only applies for women aged 25-59 in 3-year intervals [5]. The response to the limitations of Polish screening, also resulting from pandemic screening tests reduction, are the interim guidelines for cervical cancer screening, which approved the 2019 ASCCP Risk-Based Management as one of the possible screening options [18]. However, the application of the 2019 ASCCP guidelines to Polish conditions requires the fulfillment of basic conditions, including, in particular, the availability of validated diagnostic media, cytologic liquid-based preparation, HRHPV tests, standardized colposcopy, pathology infrastructure, and follow-up capabilities.

The strengths of the study include: (1) this is the first study analyzing such a large number of LBS results in Poland and Central Eastern Europe countries; (2) the large age range of the analyzed population; (3) our study provides insight into the screening tests results performed in the private-based opportunistic cervical cancer screening; (4) this is one of the largest studies presenting cytologic-virologic-immunocytochemical-histologic correlations in cervical cancer screening; (5) the time elapsed from the abnormal initial screening result and a colposcopy with a biopsy not exceeding 3 months, for an immediate histologic correlation; (6) the study shows the efficacy of QA/QC protocols for gynecologic cyto- and histopathology introduced outside of the laboratory. The limitations of the study include: (1) it is a post-hoc analysis; (2) not all patients with abnormal cytology obtained in cotesting, or primary cytology with reflex HRHPV14 testing, who had indications, decided to undergo a colposcopy with a biopsy at the Center; (3) the gynecological pathologist employed by the Center was controlled with internal QA/QC tools used outside of the laboratory performing the cytological and histopathological preparation; (4) the study showed a slightly lower positive predictive value in cytology for histologic HSIL detection $(69.46 \%)$ than that reported by CAP. Due to different colposcopic protocols and/or different histologic terminology and/or the lack of p16 stain used in cervical histologic specimens, the results of colposcopic biopsies performed outside the Center were not included in the study.

\section{Conclusions}

In conclusion, the analysis of LBC, HRHPV14, and p16/Ki67 dual-staining reporting rates with histologic correlations presented in this age-stratified study on private opportunistic cervical cancer screening, the first on such a large scale in Poland and other countries of the region, demonstrates the possibility of the effective application of a privatebased model in cervical cancer prevention. This is particularly relevant in light of the 
ineffectiveness of the organized population-based program due to low screening coverage. The data provided in our study can give a valuable benchmark, and might significantly fill the gap in Polish cervical cancer screening, not only in LBC and HRHPV14 testing, but also in p16/Ki67 dual staining. This study also showed that the new 2019 ASCCP risk-based guidelines can be reliably introduced outside the US, including in Polish private-based opportunistic secondary cervical cancer prevention, on the condition of adequate QA/QC gynecological cytopathology and colposcopy protocols being provided.

Author Contributions: Conceptualization, M.M. and M.T.; Collecting of data, K.M., Z.J., J.W., and M.P.; Data curation: K.M. and M.M.; Analysis of data, M.M. and K.M.; Interpretation of data M.T. and M.M.; Investigation, I.K.-S., M.M., M.K., M.W., A.P., P.B., and M.T.; Writing draft, M.T. and M.M.; Draft editing: M.T., K.M., and M.M.; Supervision and review R.J.; Review A.H. All authors have read and agreed to the published version of the manuscript.

Funding: This research received no external funding.

Institutional Review Board Statement: Ethical review and approval were waived for this study, due to an analysis performed that is not a clinical trial. The National Research Ethics Committee's approval was not required for this study. The process of data extraction was consistent with data protection rules in accordance with EU regulation. This post-hoc analysis shows test results in a private outpatient health center, and there was not any impact on patient management following this analysis.

Informed Consent Statement: Informed consent was obtained from all subjects involved in the study.

Data Availability Statement: The data presented in this study are available on request from the corresponding author.

Conflicts of Interest: The authors declare no conflict of interest.

\section{References}

1. Jeronimo, J.; Castle, P.E.; Temin, S.; Denny, L.; Gupta, V.; Kim, J.J.; Luciani, S.; Murokora, D.; Ngoma, T.; Qiao, Y.; et al. Secondary Prevention of Cervical Cancer: ASCO Resource-Stratified Clinical Practice Guideline. J. Glob. Oncol. 2016, 3, 635-657. [CrossRef]

2. Von Karsa, L.; Arbyn, M.; De Vuyst, H.H.; Dillner, J.; Dillner, L.; Franceschi, S.; Patnick, J.; Ronco, G.; Segnan, N.; Suonio, E.; et al. European guidelines for quality assurance in cervical cancer screening. Summary of the supplements on HPV screening and vaccination. Papillomavirus Res. 2015, 1, 22-31. [CrossRef]

3. National Cervical Cancer Screening Programme in Poland Coordinating Centre; Polish Gynaecological Society; Polish Society of Pathologists; Polish Society of Colposcopy and Uterine Cervix Pathology. Management of abnormal pap smear-Consensus guidelines of the National Cervical Cancer Screening Programme in Poland Coordinating Centre, the Polish Gynaecological Society, the Polish Society of Pathologists, and Polish Society of Colposcopy and Uterine Cervix Pathology. Ginekol. Pol. 2009, 80, 129-138. [PubMed]

4. Nowakowski, A.; Arbyn, M.; Turkot, M.H.; Wieszczy, P.; Miłosz, K.; Kamiński, M.F.; Didkowska, J.; Bidziński, M.; Olszewski, W.; Wielgoś, M.; et al. A roadmap for a comprehensive control of cervical cancer in Poland: Integration of available solutions into current practice in primary and secondary prevention. Eur. J. Cancer Prev. 2020, 29, 157-164. [CrossRef]

5. Narodowy Fundusz Zdrowia. Available online: http://www.nfz.gov.pl/dla-pacjenta/programy-profilaktyczne/ (accessed on 14 February 2021).

6. Nowakowski, A.; Cybulski, M.; Śliwczyński, A.; Chil, A.; Teter, Z.; Seroczyński, P.; Arbyn, M.; Anttila, A. The implementation of an organised cervical screening programme in Poland: An analysis of the adherence to European guidelines. BMC Cancer 2015, 15, 279, PMCID:PMC4417537. [CrossRef] [PubMed]

7. Nowakowski, A.; Wojciechowska, U.; Wieszczy, P.; Cybulski, M.; Kamiński, M.F.; Didkowska, J. Trends in cervical cancer incidence and mortality in Poland: Is there an impact of the introduction of the organised screening? Eur. J. Epidemiol. 2017, 32, 529-532. [CrossRef] [PubMed]

8. Turkot, M.; Mokwa, D.; Wieszczy, P.; Spych, K.; Kurowska, N.; Nowakowski, A.; Kamiński, M.F. External audit of providers of the cervical cancer prevention programme in Poland in 2016/2017. Nowotw. J. Oncol. 2018, 68, 65-78. [CrossRef]

9. Główny Urząd Statystyczny. Stan Zdrowia Ludności Polski w 2014 r.; Zakład Wydawnictw Statystycznych: Warszawa, Poland, 2016.

10. Laboratoryjnych, K.I.D.; Ginekologiczne, P.D. Wytyczne dotyczace aplikacji testów molekularnych identyfikujacych DNA HPV HR w profilaktyce szyjki macicy. Stanowisko ekspertów PTG i KIDL [Guidelines for application of molecular tests identyfying HR HPV DNA in the prevention of cervical cancer. Statement of experts from PGS (PTG) and NCLD (KIDL)]. Ginekol. Pol. 2013, 84, 395-399. [PubMed] 
11. Bergeron, C.; Ikenberg, H.; Sideri, M.; Denton, K.; Bogers, J.; Schmidt, D.; Alameda, F.; Keller, T.; Rehm, S.; Ridder, R. PALMS Study Group. Prospective evaluation of p16/Ki-67 dual-stained cytology for managing women with abnormal Papanicolaou cytology: PALMS study results. Cancer Cytopathol. 2015, 123, 373-381. [CrossRef] [PubMed]

12. Schmidt, D.; Bergeron, C.; Denton, K.J.; Ridder, R. European CINtec Cytology Study Group. p16/ki-67 dual-stain cytology in the triage of ASCUS and LSIL papanicolaou cytology: Results from the European equivocal or mildly abnormal Papanicolaou cytology study. Cancer Cytopathol. 2011, 119, 158-166. [CrossRef] [PubMed]

13. Wentzensen, N.; Schwartz, L.; Zuna, R.E.; Smith, K.; Mathews, C.; Gold, M.A.; Allen, R.A.; Zhang, R.; Dunn, S.T.; Walker, J.L.; et al. Performance of p16/Ki-67 immunostaining to detect cervical cancer precursors in a colposcopy referral population. Clin. Cancer Res. 2012, 18, 4154-4162, PMCID:PMC4237612. [CrossRef] [PubMed]

14. Nasierowska-Guttmejer, A.; Kędzia, W.; Wojtylak, S.; Lange, D.; Rokita, W.; Jach, R.; Wielgoś, M. Polish recommendations regarding diagnostics and treatment of cervical squamous intraepithelial lesions according to the CAP/ASCCP guidelines. Ginekol. Pol. 2016, 87, 670-676. [CrossRef] [PubMed]

15. U.S. Food and Drug Administration. Premarket Approval. Available online: https://www.accessdata.fda.gov/scripts/cdrh/ cfdocs / cfpma/pma.cfm?id=P190024 (accessed on 1 April 2020).

16. Perkins, R.B.; Guido, R.S.; Castle, P.E.; Chelmow, D.; Einstein, M.H.; Garcia, F.; Huh, W.K.; Kim, J.J.; Moscicki, A.B.; Nayar, R.; et al. 2019 ASCCP Risk-Based Management Consensus Guidelines Committee. 2019 ASCCP Risk-Based Management Consensus Guidelines for Abnormal Cervical Cancer Screening Tests and Cancer Precursors. J. Low Genit. Tract. Dis. 2020, 24, 102-131, PMCID:PMC7147428. [CrossRef] [PubMed]

17. Jach, R.; Mazurec, M.; Trzeszcz, M.; Bartosinska-Dyc, A.; Galarowicz, B.; Kedzia, W.; Nowakowski, A.; Pitynski, K. COLPOSCOPY 2020-COLPOSCOPY PROTOCOLS: A Summary of the Clinical Experts Consensus Guidelines of the Polish Society of Colposcopy and Cervical Pathophysiology and the Polish Society of Gynaecologists and Obstetricians. Ginekol. Pol. 2020, 91, 362371. [CrossRef] [PubMed]

18. Jach, R.; Mazurec, M.; Trzeszcz, M.; Zimmer, M.; Kedzia, W.; Wolski, H. Cervical cancer screening in Poland in current SARS-CoV2 pandemic: Interim guidelines of the Polish Society of Gynecologists and Obstetricians and the Polish Society of Colposcopy and Cervical Pathophysiology-A summary January 2021. Ginekol. Pol. 2021, 92, 165-173. [CrossRef] [PubMed]

19. Loghavi, S.; Walts, A.E.; Bose, S. CINtec ${ }^{\circledR}$ PLUS dual immunostain: A triage tool for cervical pap smears with atypical squamous cells of undetermined significance and low grade squamous intraepithelial lesion. Diagn. Cytopathol. 2013, 41, 582-587. [CrossRef] [PubMed]

20. Eversole, G.M.; Moriarty, A.T.; Schwartz, M.R.; Clayton, A.C.; Souers, R.; Fatheree, L.A.; Chmara, B.A.; Tench, W.D.; Henry, M.R.; Wilbur, D.C. Practices of participants in the college of american pathologists interlaboratory comparison program in cervicovaginal cytology, 2006. Arch. Pathol. Lab Med. 2010, 134, 331-335. [CrossRef] [PubMed]

21. Davey, D.D.; Souers, R.J.; Goodrich, K.; Mody, D.R.; Tabbara, S.O.; Booth, C.N. Bethesda 2014 Implementation and Human Papillomavirus Primary Screening: Practices of Laboratories Participating in the College of American Pathologists PAP Education Program. Arch. Pathol. Lab Med. 2019, 143, 1196-1202. [CrossRef] [PubMed]

22. Crothers, B.A.; Jones, B.A.; Cahill, L.A.; Moriarty, A.T.; Mody, D.R.; Tench, W.D.; Souers, R.J. Quality improvement opportunities in gynecologic cytologic-histologic correlations: Findings from the College of American Pathologists Gynecologic Cytopathology Quality Consensus Conference working group 4. Arch. Pathol. Lab Med. 2013, 137, 199-213. [CrossRef] [PubMed]

23. Tavares, S.B.; de Souza, N.L.; Manrique, E.J.; Azara, C.Z.; da Silveira, E.A.; Amaral, R.G. Internal quality control for cervical cytopathology: Comparison of potential false-negatives detected at rapid prescreening and at $100 \%$ rapid review. Acta. Cytol. 2014, 58, 439-445. [CrossRef] [PubMed]

24. Nayar, R.; Wilbur, D.C. The Bethesda System for Reporting Cervical Cytology-Definitions, Criteria, and Explanatory Notes; Springer International Publishing: Cham, Switzerland, 2015. [CrossRef]

25. Massad, L.S.; Einstein, M.H.; Huh, W.K.; Katki, H.A.; Kinney, W.K.; Schiffman, M.; Solomon, D.; Wentzensen, N.; Lawson, H.W. 2012 ASCCP Consensus Guidelines Conference. 2012 updated consensus guidelines for the management of abnormal cervical cancer screening tests and cancer precursors. J. Low Genit. Tract. Dis. 2013, 17, S1-S27. [CrossRef] [PubMed]

26. Huh, W.K.; Ault, K.A.; Chelmow, D.; Davey, D.D.; Goulart, R.A.; Garcia, F.A.R.; Kinney, W.K.; Massad, L.S.; Mayeaux, E.J.; Saslow, D.; et al. Use of primary high-risk human papillomavirus testing for cervical cancer screening: Interim clinical guidance. Obstet. Gynecol. 2015, 125, 330-337. [CrossRef] [PubMed]

27. Darragh, T.M.; Colgan, T.J.; Cox, J.T.; Heller, D.S.; Henry, M.R.; Luff, R.D.; McCalmont, T.; Nayar, R.; Palefsky, J.M.; Stoler, M.H.; et al. Members of LAST Project Work Groups. The Lower Anogenital Squamous Terminology Standardization Project for HPV-Associated Lesions: Background and consensus recommendations from the College of American Pathologists and the American Society for Colposcopy and Cervical Pathology. Arch. Pathol. Lab Med. 2013, 136, 1266-1297. [CrossRef] [PubMed]

28. Kurman, R.J.; Carcangiu, M.L. WHO Classification of Tumours of Female Reproductive Organs, 4th ed.; International Agency for Research on Cancer (IARC): Lyon, France, 2014.

29. IARC Working Group on the Evaluation of Carcinogenic Risks to Humans. Biological agents. Volume 100 B. A review of human carcinogens. IARC Monogr. Eval. Carcinog. Risks Hum. 2012, 100, 1-441, PMCID:PMC4781184. [PubMed]

30. De Sanjosé, S.; Diaz, M.; Castellsagué, X.; Clifford, G.; Bruni, L.; Muñoz, N.; Bosch, F.X. Worldwide prevalence and genotype distribution of cervical human papillomavirus DNA in women with normal cytology: A meta-analysis. Lancet Infect. Dis. 2007, 7, 453-459. [CrossRef] [PubMed] 
31. Zhao, C.; Moriarty, A.T.; Ghofrani, M.; Husain, M.; Tambouret, R.H.; Laucirica, R.; Laser, A.; Fischer, A.; Ocal, I.T.; Souers, R.J.; et al. Human papillomavirus testing and reporting rates in 2012: Results of a College of American Pathologists national survey. Arch. Pathol. Lab Med. 2015, 139, 757-761. [CrossRef] [PubMed]

32. Arbyn, M.; Martin-Hirsch, P.; Buntinx, F.; Van Ranst, M.; Paraskevaidis, E.; Dillner, J. Triage of women with equivocal or low-grade cervical cytology results: A meta-analysis of the HPV test positivity rate. J. Cell Mol. Med. 2009, 13, 648-659, PMCID:PMC3822872. [CrossRef] [PubMed]

33. Tao, X.; Zhang, H.; Wang, L.; Pan, Q.; Ji, S.; Zhou, X.; Zhao, C. Atypical squamous cells of undetermined significance cervical cytology in the Chinese population: Age-stratified reporting rates, high-risk HPV testing, and immediate histologic correlation results. Cancer Cytopathol. 2021, 129, 24-32. [CrossRef] [PubMed]

34. Tao, X.; Zhang, H.; Wang, S.; Chen, T.; Cong, Q.; Wang, L.; Zhou, X.; Zhao, C. Prevalence and carcinogenic risk of high-risk human papillomavirus subtypes in different cervical cytology: A study of 124,251 cases from the largest academic center in China. J. Am. Soc. Cytopathol. 2021, 10, 391-398. [CrossRef] [PubMed]

35. Demarco, M.; Lorey, T.S.; Fetterman, B.; Cheung, L.C.; Guido, R.S.; Wentzensen, N.; Kinney, W.K.; Poitras, N.E.; Befano, B.; Castle, P.E.; et al. Risks of CIN 2+, CIN 3+, and Cancer by Cytology and Human Papillomavirus Status: The Foundation of Risk-Based Cervical Screening Guidelines. J. Low Genit. Tract. Dis. 2017, 21, 261-267, PMCID:PMC5625966. [CrossRef] [PubMed] 
36. Wheeler, C.M.; Hunt, W.C.; Cuzick, J.; Langsfeld, E.; Pearse, A.; Montoya, G.D.; Robertson, M.; Shearman, C.A.; Castle, P.E.; New Mexico HPV Pap Registry Steering Committee. A population-based study of human papillomavirus genotype prevalence in the United States: Baseline measures prior to mass human papillomavirus vaccination. Int. J. Cancer 2013, 132, 198-207. [CrossRef] [PubMed]

37. Tao, X.; Zhang, H.; Zhang, H.; Xiao, J.; Li, J.; Zhou, X.; Wang, L.; Zhao, C. Follow-up with histopathology and HPV testing on LSIL cytology in China's largest academic woman's hospital. Cancer Cytopathol. 2019, 127, 258-266. [CrossRef] [PubMed]

38. Tao, X.; Austin, R.M.; Zhang, H.; Zhang, L.; Xiao, J.; Zhou, X.; Wang, L.; Zhao, C. Histopathologic follow-up and HPV test results with HSIL Papanicolaou test results in China's largest academic women's hospital. Cancer Cytopathol. 2017, 125, 947-953. [CrossRef] [PubMed]

39. Ronco, G.; Giorgi-Rossi, P.; Carozzi, F.; Dalla Palma, P.; Del Mistro, A.; De Marco, L.; De Lillo, M.; Naldoni, C.; Pierotti, P.; Rizzolo, R.; et al. New Technologies for Cervical Cancer screening Working Group. Human papillomavirus testing and liquid-based cytology in primary screening of women younger than 35 years: Results at recruitment for a randomised controlled trial. Lancet Oncol. 2006, 7, 547-555. [CrossRef] [PubMed]

40. Bergeron, C.; Giorgi-Rossi, P.; Cas, F.; Schiboni, M.L.; Ghiringhello, B.; Dalla Palma, P.; Minucci, D.; Rosso, S.; Zorzi, M.; Naldoni, C.; et al. Informed cytology for triaging HPV-positive women: Substudy nested in the NTCC randomized controlled trial. J. Natl. Cancer Inst. 2015, 107, 423, PMCID:PMC4339260. [CrossRef] [PubMed]

41. Wright, T.C., Jr.; Behrens, C.M.; Ranger-Moore, J.; Rehm, S.; Sharma, A.; Stoler, M.H.; Ridder, R. Triaging HPV-positive women with p16/Ki-67 dual-stained cytology: Results from a sub-study nested into the ATHENA trial. Gynecol. Oncol. 2017, 144, 51-56. [CrossRef] [PubMed]

42. Ebisch, R.M.; van der Horst, J.; Hermsen, M.; Rijstenberg, L.L.; Vedder, J.E.; Bulten, J.; Bosgraaf, R.P.; Verhoef, V.M.; Heideman, D.A.; Snijders, P.J.; et al. Evaluation of p16/Ki-67 dual-stained cytology as triage test for high-risk human papillomavirus-positive women. Mod. Pathol. 2017, 30, 1021-1031. [CrossRef] [PubMed]

43. Killeen, J.L.; Dye, T.; Grace, C.; Hiraoka, M. Improved abnormal Pap smear triage using cervical cancer biomarkers. J. Low Genit. Tract. Dis. 2014, 18, 1-7. [CrossRef] [PubMed]

44. Donà, M.G.; Vocaturo, A.; Giuliani, M.; Ronchetti, L.; Rollo, F.; Pescarmona, E.; Carosi, M.; Vocaturo, G.; Benevolo, M. p16/Ki-67 dual staining in cervico-vaginal cytology: Correlation with histology, Human Papillomavirus detection and genotyping in women undergoing colposcopy. Gynecol. Oncol. 2012, 126, 198-202. [CrossRef] [PubMed]

45. OECD. Screening, Survival and Mortality for Cervical Cancer. In Health at a Glance: Europe 2018: State of Health in the EU Cycle; OECD Publishing: Paris, France, 2018. [CrossRef] 